\title{
Electronic structure and magnetic properties of the $\mathrm{Ce}_{2} \mathrm{MnGe}_{6}$ compound
}

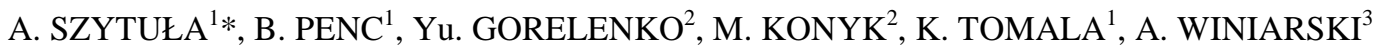 \\ ${ }^{1}$ M. Smoluchowski Institute of Physics, Jagiellonian University, Reymonta 4, 30-059 Kraków, Poland \\ ${ }^{2}$ Department of Inorganic Chemistry, Ivan Franko National University of Lviv, \\ Kyryla i Mefodiya 6, UA-79005 Lviv, Ukraine \\ ${ }^{3}$ A. Chetkowski Institute of Physics, University of Silesia, Uniwersytecka 4, 40-007 Katowice, Poland \\ * Corresponding author. E-mail address: szytula@if.uj.edu.pl
}

Received February 15, 2008; accepted February 17, 2008; available on-line March 31, 2008

\begin{abstract}
Magnetization, magnetic susceptibility and $\mathrm{X}$-ray photoemission measurements were performed on a $\mathrm{Ce}_{2} \mathrm{MnGe}_{6}$ polycrystalline sample. This compound crystallizes in an orthorhombic $\mathrm{Ce}_{2} \mathrm{CuGe}_{6}$-type structure (space group Amm2). The Ce atoms occupy two nonequivalent sites, while the Mn atoms only one site. The magnetic data indicate that the compound is a ferrimagnet with the Curie temperature $T_{\mathrm{C}}=150 \mathrm{~K}$. The magnetic moment determined from the magnetization curve measured at $2 \mathrm{~K}$ increases from $1.9 \mu_{\mathrm{B}}$ for $H \approx 0$ to $2.5 \mu_{\mathrm{B}}$ for $H=50 \mathrm{kOe}$. Above $T_{\mathrm{C}}$ the reciprocal magnetic susceptibility fulfills the Curie-Weiss law with the effective magnetic moment equal $5.3 \mu_{\mathrm{B}}$. The valence band gives a small peak at $0.3 \mathrm{eV}$ below the Fermi level and a broad one with the maximum at $2.1 \mathrm{eV}$, corresponding to the Mn 3d states. The structure of the Ce $3_{5 / 2}$ and Ce $3 d_{3 / 2}$ XPS spectra has been interpreted in terms of the Gunnarsson-Schönhammer theory. Two final-state contributions of $\mathrm{f}^{1}$ and $\mathrm{f}^{2}$, exhibiting a spin-orbit splitting of $\Delta_{\mathrm{SO}}=18.7 \mathrm{eV}$, were clearly observed. From the intensity ratio $r=I\left(f^{2}\right) /\left[I\left(f^{1}\right)+I\left(f^{2}\right)\right]=0.20$ a hybridization energy equal $93 \mathrm{eV}$ was determined.
\end{abstract}

Ternary rare earth germanides / Magnetic ordering / Electronic structure

\section{Introduction}

This investigation is part of a broader study, which is expected to systematize the magnetic properties of the $\mathrm{R}_{\mathrm{m}} \mathrm{T}_{\mathrm{n}} \mathrm{X}_{\mathrm{p}}$ intermetallic compounds, where $\mathrm{R}$ is a rareearth atom, $\mathrm{T}$ is a d-electron atom and $\mathrm{X}$ is a p-electron atom. The compound investigated in this work belongs to the $\mathrm{R}_{2} \mathrm{TGe}_{6}$ family, where $\mathrm{T}$ is $\mathrm{Mn}$, $\mathrm{Cu}$. These compounds crystallize in a orthorhombic crystal structure of the $\mathrm{Ce}_{2} \mathrm{CuGe}_{6}$ type (space group Amm2) [1,2]. The isostructural $\mathrm{R}_{2} \mathrm{CuGe}_{6}(\mathrm{R}=\mathrm{Gd}, \mathrm{Tb}$, Dy, Er) compounds order antiferromagnetically at low temperatures with the Néel temperature between $5.6 \mathrm{~K}$ (for $\mathrm{R}=\mathrm{Er}$ ) and $33.1 \mathrm{~K}$ (for $\mathrm{R}=\mathrm{Tb}$ ) [3].

In this work we report results of magnetic and XPS measurements for $\mathrm{Ce}_{2} \mathrm{MnGe}_{6}$. From these data magnetic properties and the electronic structure were determined.

\section{Experimental details}

The sample was prepared by arc-melting the elements (cerium, purity 99.85 wt.\%; manganese, purity 99.99 wt.\%; germanium purity 99.99 wt.\%) under a high-purity Ti-gettered argon atmosphere on a watercooled copper hearth. The alloy was then annealed at $670 \mathrm{~K}$ in an evacuated quartz glass tube for 4 weeks, and subsequently quenched into cold water.

The quality of the sample was checked by X-ray diffraction performed at room temperature on powder samples using a DRON-3.0 diffractometer, $\mathrm{CuK}_{\alpha}$ radiation. All the procedures, including indexing, refinement of the lattice and atomic parameters, were accomplished using the CSD program package [4].

DC magnetic measurements were carried out using a commercial MPMS SQUID magnetometer. Two types of measurement were performed: magnetic susceptibility measurements in a magnetic field of $1 \mathrm{kOe}$ in the temperature range $2-300 \mathrm{~K}$ (from these data the effective magnetic moment $\mu_{\text {eff }}$ and paramagnetic Curie temperature $\theta_{\mathrm{p}}$ were obtained) and magnetization measurements in magnetic fields up to $50 \mathrm{kOe}$ at about $2 \mathrm{~K}$ (in order to get the value of the pseudo-saturated magnetic moment and the character of the magnetization curve). Since the crystallites had not well defined shapes, no demagnetization effects were taken into account.

XPS spectra were obtained at room temperature using a Leybold LHS10 electron photoemission 
spectrometer with $\mathrm{MgK}_{\alpha}(\mathrm{h} v=1253.6 \mathrm{eV})$ and $\mathrm{AlK}_{\alpha}$ $(\mathrm{h} v=1486.6 \mathrm{eV})$ radiation in a vacuum of about $10^{-9}$ mbar. The total energy resolution of the spectrometer with a hemispherical energy analyzer was about $0.75 \mathrm{eV}$ for $\mathrm{Ag} 3 \mathrm{~d}$. Binding energies were referred to the Fermi level $\left(\mathrm{E}_{\mathrm{F}}=0\right)$. The spectrometer was calibrated using $\mathrm{Cu} 2 \mathrm{p}_{3 / 2}(932.5 \mathrm{eV}), \mathrm{Ag} 3 \mathrm{~d}_{5 / 2}$ $(368.1 \mathrm{eV})$ and $\mathrm{Au} \quad 4 \mathrm{f}_{7 / 2}(84.0 \mathrm{eV})$ core-level photoemission spectra. The surface of the sample was mechanically cleaned by scraping with a diamond file in a preparation chamber under high vacuum $\left(10^{-9} \mathrm{mbar}\right)$, and then moved immediately into the analysis chamber. This procedure was repeated until the $\mathrm{C} 1 \mathrm{~s}$ and $\mathrm{O} 1 \mathrm{~s}$ core-level peaks were negligibly small, or were not changed after further scraping. This cleaning procedure was performed before each XPS measurement. The Shirley method [5] was used to subtract the background and then the experimental spectra were numerically fitted using a $80 \%$ Gaussian and $20 \%$ Lorentzian model.

\section{Results}

The investigated $\mathrm{Ce}_{2} \mathrm{MnGe}_{6}$ sample has an orthorhombic crystal structure of the $\mathrm{Ce}_{2} \mathrm{CuGe}_{6}$ type. The lattice parameters refined to $a=0.4103(5) \mathrm{nm}$, $b=0.4342(5) \mathrm{nm}$ and $c=2.188(1) \mathrm{nm}$. Fig. 1 shows the temperature dependence of the magnetic moment and of the reciprocal magnetic susceptibility of $\mathrm{Ce}_{2} \mathrm{MnGe}_{6}$ between 2 and $300 \mathrm{~K}$. In the temperature range $2-150 \mathrm{~K}$ a spontaneous magnetic moment exists. Above $150 \mathrm{~K}$ the reciprocal magnetic susceptibility obeys the Curie-Weiss law with an effective magnetic moment equal $5.3 \mu_{\mathrm{B}}$ for the formula unit and a paramagnetic Curie temperature equal $+150 \mathrm{~K}$. The magnetization curve measured at $2 \mathrm{~K}$ in fields up to $50 \mathrm{kOe}$ (inset of Fig. 1) shows the ferromagnetic character. A sharp jump of the magnetization at low field, followed by an increase $\left(0.012 \mu_{\mathrm{B}} / \mathrm{kOe}\right)$ with increasing magnetic field, is observed. The magnetic moment at $2 \mathrm{~K}$ in a magnetic field of $50 \mathrm{kOe}$ is $2.5 \mu_{\mathrm{B}}$.

The XPS valence band spectrum extending from the Fermi energy level located at $0 \mathrm{eV}$ to a binding energy of about $15 \mathrm{eV}$ is presented in Fig. 2. The valence band is dominated by a broad maximum at $2.1 \mathrm{eV}$ corresponding to the Mn 3d states. Near the Fermi level, at $0.3 \mathrm{eV}$, a small peak corresponding to the $\mathrm{Ce} 4 \mathrm{f}$ state is observed. The peaks at 6.6 and $9.7 \mathrm{eV}$ correspond to the $\mathrm{Ge} 4 \mathrm{~s}$ and $4 \mathrm{p}$ states. The $\mathrm{Ce}$ 4f and Mn 3d states overlap. In order to obtain information on the electronic structure of the $\mathrm{Ce}$ atoms, the $\mathrm{Ce} 4 \mathrm{~d}$ and $\mathrm{Ce} 3 \mathrm{~d}$ states were analyzed (Fig. 3). For both states the spin-orbit splitting dominates in the spectral structure and is equal $2.8 \mathrm{eV}$ for the Ce $4 \mathrm{~d}$ states and $18.7 \mathrm{eV}$ for the Ce $3 \mathrm{~d}$ states. This splitting is typical for cerium intermetallic compounds. For both states the spin-orbit splitting dominates in the spectral structure and is equal $2.8 \mathrm{eV}$ for the Ce $4 \mathrm{~d}$ states and $18.7 \mathrm{eV}$ for the Ce $3 \mathrm{~d}$ states.

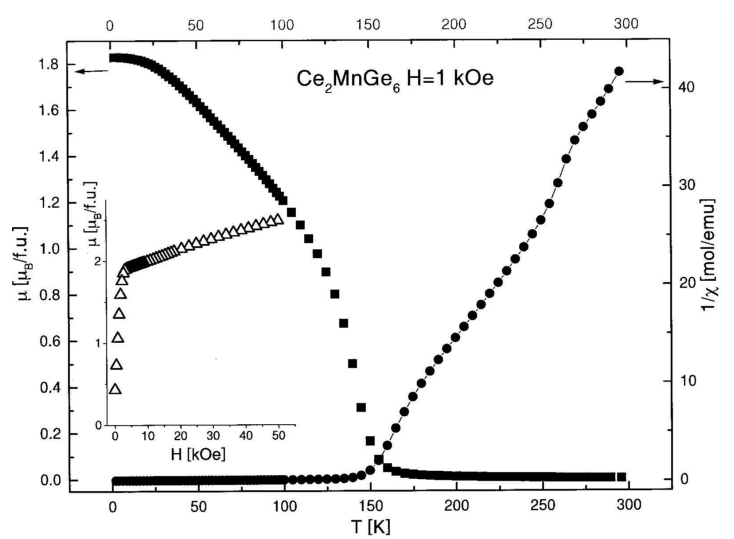

Fig. 1 Temperature dependence of the magnetic moment and the reciprocal magnetic susceptibility of $\mathrm{Ce}_{2} \mathrm{MnGe}_{6}$ in an external magnetic field equal $1 \mathrm{kOe}$. The inset shows the magnetization curve at $2 \mathrm{~K}$.

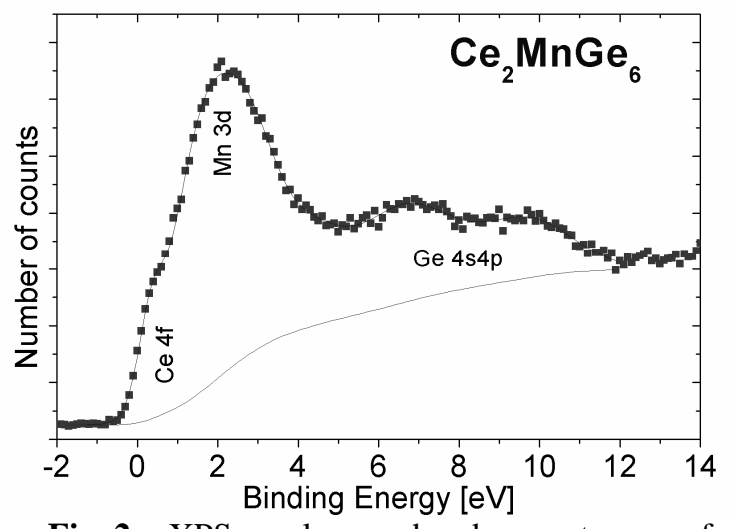

Fig. 2 XPS valence band spectrum of $\mathrm{Ce}_{2} \mathrm{MnGe}_{6}$.

This splitting is typical for cerium intermetallic compounds. On the low-binding-energy side of the $3 d_{5 / 2}$ and $3 d_{3 / 2}$ main lines shake-down satellites are observed. The separation of these peaks is explained by the Doniach-Šunjić theory [6], which yields the intensity ratio $\mathrm{r}=\mathrm{I}\left(\mathrm{f}^{2}\right) /\left[\mathrm{I}\left(\mathrm{f}^{1}\right)+\mathrm{I}\left(\mathrm{f}^{2}\right)\right]$. On the basis of the Gunnarsson-Schönhammer model [7] it was possible to determine the hybridization energy $\Delta$ between the $4 \mathrm{f}$ states and the conduction electrons. The parameter $\Delta$ is defined as $\pi \mathrm{V}^{2} \rho_{\max }$, where $\mathrm{V}$ is the hybridization matrix element and $\rho_{\max }$ is the maximum of the density of states of the conduction band. The $r$ value is equal 0.2 and the corresponding hybridization energy is $93 \mathrm{meV}$. The absence of a peak corresponding to the $\mathrm{Ce} 4 \mathrm{f}^{0}$ state indicates that cerium is present as trivalent ions.

The Mn 2p states form band peaks with a spinorbit splitting equal $11.4 \mathrm{eV}$ and a complex structure, which is caused by different atomic surroundings and oxidation (Fig. 4).

The analysis of the Ge $3 p$ and Ge $2 p$ states indicates that the values of the binding energy and the spin-orbit splittings $4.0 \mathrm{eV}$ for the Ge $3 \mathrm{p}$ states and $31.0 \mathrm{eV}$ for $\mathrm{Ge} 2 \mathrm{p}$ states are near the values of the pure element given in [8]. 

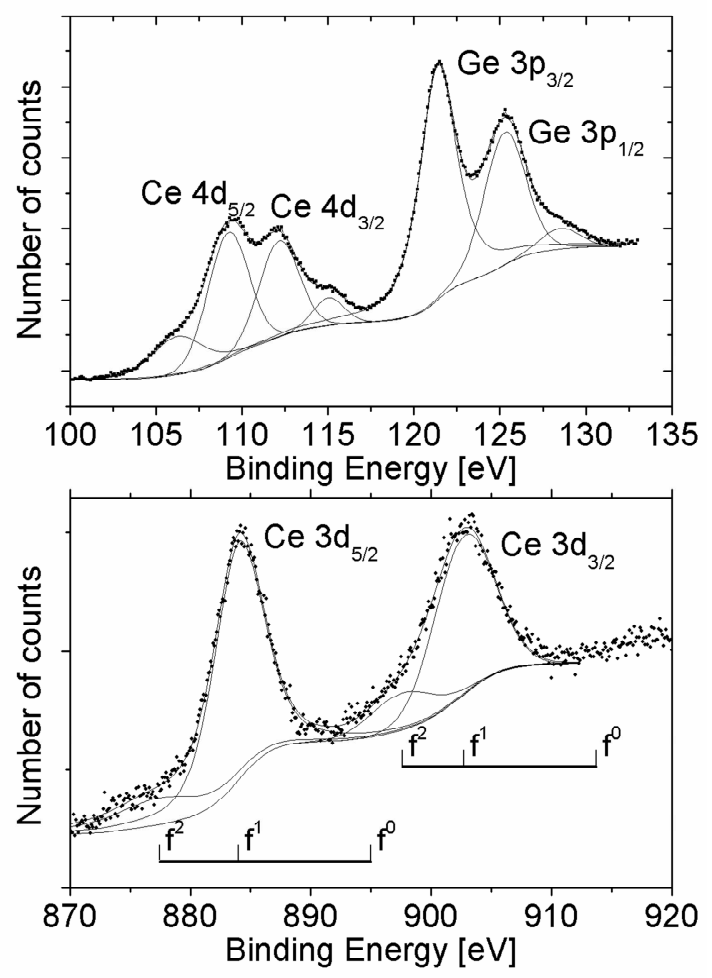

Fig. 3 (a) Ce $4 d_{5 / 2}$, Ce $4 d_{3 / 2}, G e 3 p_{3 / 2}$ and Ge $3 \mathrm{p}_{1 / 2}$, and (b) $\mathrm{Ce} 3 \mathrm{~d}_{5 / 2}$ and $\mathrm{Ce} 3 \mathrm{~d}_{3 / 2}$ XPS spectra of $\mathrm{Ce}_{2} \mathrm{MnGe}_{6}$.

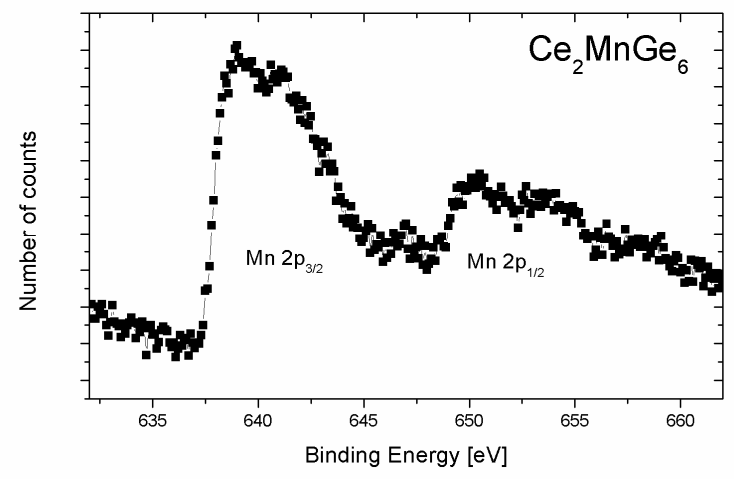

Fig. $4 \mathrm{Mn} 2 \mathrm{p}_{3 / 2}$ and $\mathrm{Mn} 2 \mathrm{p}_{1 / 2}$ spectrum of $\mathrm{Ce}_{2} \mathrm{MnGe}_{6}$.

\section{Conclusions}

The results presented in this work indicate that $\mathrm{Ce}_{2} \mathrm{MnGe}_{6}$ is a ferrimagnet with a Curie temperature of $150 \mathrm{~K}$. A comparison of the value of the critical temperature of the magnetic order of $\mathrm{Ce}_{2} \mathrm{CuGe}_{6}$ with those observed for other $\mathrm{R}_{2} \mathrm{CuGe}_{6}$ compounds [3], and the high value of the Curie temperature of $\mathrm{Ce}_{2} \mathrm{MnGe}_{6}$, indicate that the Mn magnetic moments order in the latter. The field dependence of the magnetization suggests a complex ferrimagnetic ordering.

The XPS data indicate that cerium is trivalent and that the hybridization of the $\mathrm{Ce} 4 \mathrm{f}$ states with electrons of the conduction band is weak. Assuming a tripositive cerium ion, the effective manganese moment is $3.9 \mu_{\mathrm{B}}$. The value of the magnetic moment in the ordered state equals $2.5 \mu_{\mathrm{B}}$, indicating a ferri- or complex magnetic structure.
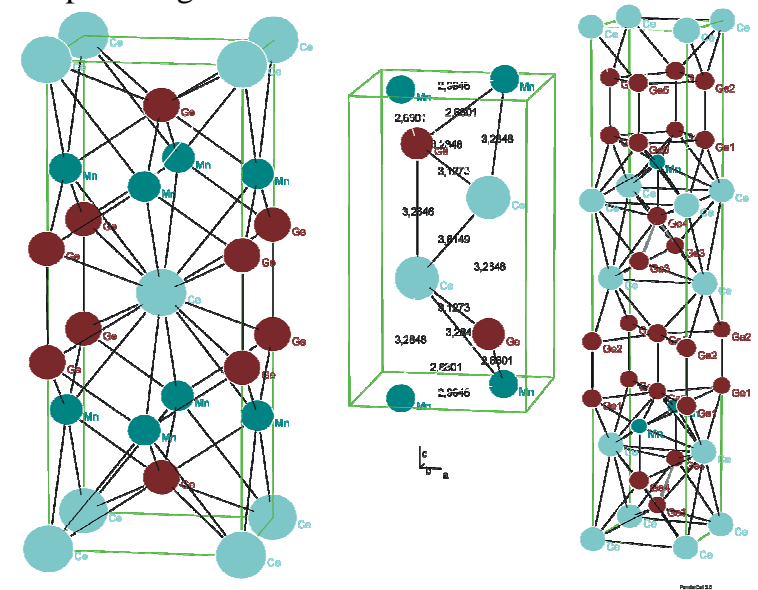

Fig. 5 Crystal structures of (a) $\mathrm{CeMn}_{2} \mathrm{Ge}_{2}$, (b) CeMnGe and (c) $\mathrm{Ce}_{2} \mathrm{MnGe}_{6}$.

Two other compounds in the Ce-Mn-Ge ternary system have different magnetic structures. The CeMnGe and $\mathrm{CeMn}_{2} \mathrm{Ge}_{2}$ compounds have tetragonal crystal structures of the CeFeSi type (space group $P 4 / n m m$ ) and $\mathrm{CeAl}_{2} \mathrm{Ga}_{2}$ type (space group $\mathrm{I} / \mathrm{mmm}$ ), respectively. The crystal structures of these compounds and of $\mathrm{Ce}_{2} \mathrm{MnGe}_{6}$ are shown in Fig. 5. In CeMnGe below $T_{\mathrm{t}}=130 \mathrm{~K}$ both the Ce and the $\mathrm{Mn}$

Table 1 Values of $\Delta_{i j}=d_{i j}-\left(R_{i}+R_{j}\right)$ for CeMnGe, CeMn ${ }_{2} \mathrm{Ge}_{2}$ and $\mathrm{Ce}_{2} \mathrm{MnGe}_{6} ; \mathrm{d}_{\mathrm{ij}}$ is the interatomic distance between the atoms $i$ and $j, R_{i}$ and $R_{j}$ are their atomic radii.

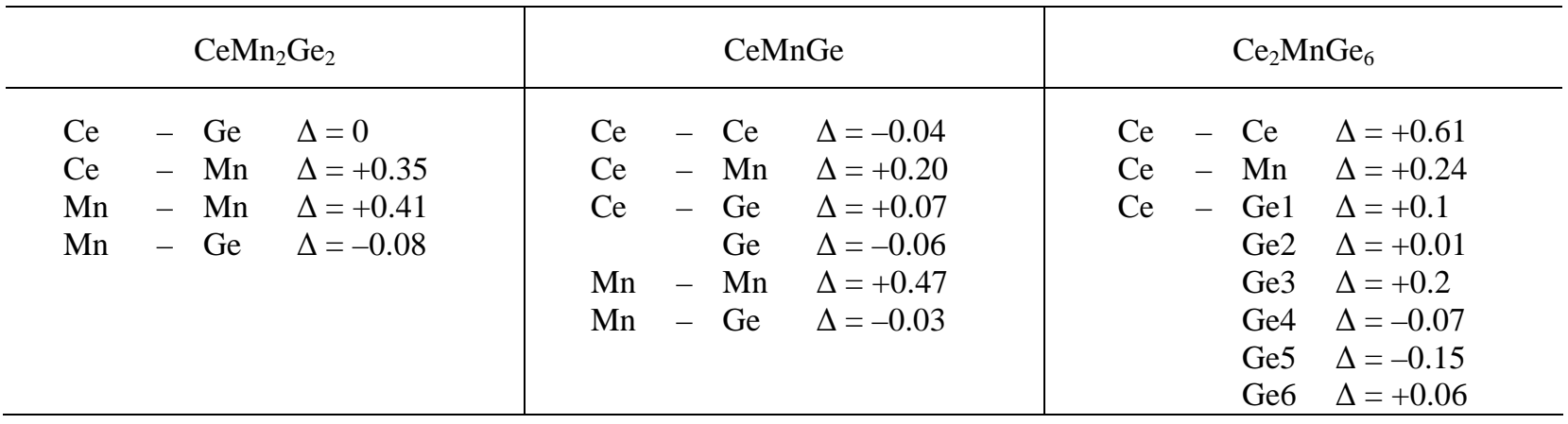


moments form collinear antiferromagnetic structures. At $2 \mathrm{~K}$ the moments of $\mathrm{Ce}$ and $\mathrm{Mn}$ are equal 2.24(6) $\mu_{\mathrm{B}}$ and 3.15(4) $\mu_{\mathrm{B}}$, respectively. Above $130 \mathrm{~K}$ up to the Néel temperature equal $532 \mathrm{~K}$ only the Mn moments order [9].

In the temperature range $2-300 \mathrm{~K}, \mathrm{CeMn}_{2} \mathrm{Ge}_{2}$ $\left(T_{\mathrm{C}}=320 \mathrm{~K}\right)$ exhibits an easy axis conical magnetic structure with a total Mn moment of about $2.7 \mu_{\mathrm{B}}$ at $2 \mathrm{~K}$. The $\mathrm{Ce}$ magnetic moments do not order above $1.6 \mathrm{~K}[10]$.

These results indicate that the magnetic ordering changes with the crystal structure and the concentration of $\mathrm{Ce}$ and $\mathrm{Mn}$ atoms. A comparison of the interatomic distances in the three compounds discussed above with the sum of atomic radii is given in Table 1.

Neutron diffraction experiments are necessary to explain the magnetic properties of $\mathrm{Ce}_{2} \mathrm{MnGe}_{6}$ at low temperatures.

\section{References}

[1] M.B. Konyk, P.S. Salamakha, O.I. Bodak, V.K. Pecharsky, Kristallografia 33 (1988) 838.

[2] M.B. Konyk, O.I. Bodak, J. Alloys Compd. 387 (2005) 243.

[3] D. Kaczorowski, M.B. Konyk, A. Szytuła, L. Romaka, O. Bodak, J. Alloys Compd. (this volume).

[4] L.G. Aksel'rud, Yu.N. Grin, P.Yu. Zavalij, V.K. Pecharsky, V.S. Fundamensky, XII Eur. Crystallogr. Meet., Coll. Abstr., Moscow, 1989, p. 155.

[5] D.A. Shirley, Phys. Rev. B 5 (1975) 4709.

[6] S. Doniach, M. Šujić, J. Phys. C 3 (1970) 285.

[7] O. Gunnarsson, K. Schönhammer, Phys. Rev. B 50 (1994) 12332.

[8] S. Hüfner, Photoemission Spectroscopy, Springer-Verlag, Berlin, 1994, p. 453.

[9] R. Welter, Thesis, University de Nancy I, 1994.

[10] R. Welter, G. Venturini, E. Ressouche, B. Malaman, J. Alloys Compd. 218 (1995) 204.

Proceeding of the IX International Conference on Crystal Chemistry of Intermetallic Compounds, Lviv, September 20-24, 2005. 